\title{
Death and renal transplantation among Aboriginal people undergoing dialysis
}

\author{
Marcello Tonelli, Brenda Hemmelgarn, Braden Manns, George Pylypchuk, Clara Bohm, \\ Karen Yeates, Sita Gourishankar, John S. Gill
}

ß See related articles pages 585, 597

Abstract

Background: Despite the increase in the number of Aboriginal people with end-stage renal disease around the world, little is known about their health outcomes when undergoing renal replacement therapy. We evaluated differences in survival and rate of renal transplantation among Aboriginal and white patients after initiation of dialysis.

Methods: Adult patients who were Aboriginal or white and who commenced dialysis in Alberta, Saskatchewan or Manitoba between Jan. 1, 1990, and Dec. 31, 2000, were recruited for the study and were followed until death, transplantation, loss to follow-up or the end of the study (Dec. 31, 2001). We used Cox proportional hazards models to examine the effect of race on patient survival and likelihood of transplant, with adjustment for potential confounders.

Results: Of the 4333 adults who commenced dialysis during the study period, $15.8 \%$ were Aboriginal and $72.4 \%$ were white. Unadjusted rates of death per 1000 patient-years during the study period were 158 (95\% confidence interval [Cl] 144-176) for Aboriginal patients and $146(95 \% \mathrm{Cl}$ 139-153) for white patients. When follow-up was censored at the time of transplantation, the age-adjusted risk of death after initiation of dialysis was significantly higher among Aboriginal patients than among white patients (hazard ratio [HR] 1.15, 95\% Cl 1.02-1.30). The greater risk of death associated with Aboriginal race was no longer observed after adjustment for diabetes mellitus and other comorbid conditions (adjusted HR 0.89, 95\% Cl 0.77-1.02) and did not appear to be associated with socioeconomic status. During the study period, unadjusted transplantation rates per 1000 patient-years were $62(95 \% \mathrm{Cl} 52-75)$ for Aboriginal patients and $133(95 \% \mathrm{Cl} 125-142)$ for white patients. Aboriginal patients were significantly less likely to receive a renal transplant after commencing dialysis, even after adjustment for potential confounders (HR 0.43, 95\% Cl 0.35-0.53). In an additional analysis that included followup after transplantation for those who received renal allografts, the age-adjusted risk of death associated with Aboriginal race $(\mathrm{HR} 1.36,95 \% \mathrm{Cl} 1.21-1.52)$ was higher than when follow-up after transplantation was not considered, perhaps because of the lower rate of transplantation among Aboriginals.
Interpretation: Survival among dialysis patients was similar for Aboriginal and white patients after adjustment for comorbidity. However, despite universal access to health care, Aboriginal people had a significantly lower rate of renal transplantation, which might have adversely affected their survival when receiving renal replacement therapy.

CMAJ 2004;171(6):577-82

I $\mathrm{n}$ North America and the Antipodes, the incidence of diabetes among adolescent and adult Aboriginals has risen dramatically, ${ }^{1-4}$ with corresponding increases in the prevalence of diabetic nephropathy. ${ }^{5-7}$ Aboriginal people in Canada have experienced disproportionately high incidence rates of end-stage renal disease (ESRD), with an 8fold increase in the number of prevalent dialysis patients between 1980 and 2000. ${ }^{8}$ Although the incidence of ESRD appears to have decreased in recent years, the prevalence of diabetes mellitus and its complications are rising, especially among young people. ${ }^{9-11}$

Most work evaluating health outcomes among Aboriginal people considers either the general population ${ }^{12}$ or diseases for which interventions are implemented over a short period, such as alcohol abuse, ${ }^{13}$ injury ${ }^{14}$ or critical illness. ${ }^{15}$ Death and markers of poor health are significantly more common among Aboriginal people than among North Americans of European ancestry, perhaps because of the greater prevalence of diabetes mellitus, adverse health effects due to lower socioeconomic status ${ }^{16}$ and reduced access to primary care. ${ }^{17}$ Aboriginal patients may also face unique barriers to care, including mistrust of non-Aboriginal providers, institutional discrimination or preference for traditional remedies. ${ }^{18}$ These factors may be most relevant when contact with physicians is infrequent, which obstructs development of a therapeutic relationship. In contrast, ESRD is a chronic illness that requires ongoing care from a relatively small, stable multidisciplinary team.

Although recent evidence highlights racial inequalities in morbidity and mortality among North Americans with ESRD, most studies have focused on black or Hispanic populations. ${ }^{19}$ We conducted this study to evaluate rates 
of death and renal transplantation among Aboriginal people after initiation of dialysis in Alberta, Saskatchewan and Manitoba.

\section{Methods}

We studied all adult patients who commenced dialysis treatment in Alberta, Saskatchewan and Manitoba between Jan. 1, 1990, and Dec. 31, 2000. We restricted the study to these provinces (where most of the Aboriginal population is from the Cree, Ojibway, Seaulteux and Blackfoot tribes) to increase the homogeneity of the study population. We included only patients treated in centres with kidney transplant programs because differential access to transplantation might affect survival when undergoing dialysis.
Anonymized data were provided by the sole national population-based organ failure registry in Canada, the Canadian Organ Replacement Registry (CORR). ${ }^{20,21}$ Patient-specific data, including demographic characteristics, initial mode of dialysis and comorbid conditions at the time of first dialysis treatment, are collected by health care professionals (on the basis of personal knowledge of the patient, chart review and, in some cases, direct inquiry) and are submitted annually to CORR by all Canadian dialysis centres. Changes in treatment modality (including transplantation) and dates of allograft failure and death are also recorded.

In CORR, patient race is categorized as white, black, Aboriginal, Asian, Indian subcontinent, Mideast/Arabian, Pacific Islander, other or unknown. The CORR instructions for data collection define Aboriginal race as people of "First Nations, Inuit, or Métis" origin. Race is determined at the discretion of the health care professional, since CORR procedure does not require that patients be asked directly about racial status. Because the focus of this study was on people of Aboriginal race and because nonwhite, non-Aboriginal people requiring renal replacement therapy have lower rates of death than white people,$^{22}$ the analysis presented here includes only comparisons between white and Aboriginal subjects.

Differences between the groups were compared with $\chi^{2}$ tests for categorical variables and $t$ tests for continuous variables. We estimated socioeconomic status using the neighbourhood income per person equivalent (IPPE), a measure of household income that is adjusted for the size of the household, based on summary data from the 1996 Canadian census. Within each community, the average IPPE was used to rank all subcommunities, and then the population was divided into approximate fifths to create community-specific income quintiles. ${ }^{23}$ Community size, also obtained from census data, was used as a surrogate for urban or rural location of residence and was characterized by a 4-category variable ranging from small towns with fewer than 10000 people to large urban centres with more than 500000 people.

Patients were followed from initiation of dialysis until death, transplantation, loss to follow-up or the end of the study (Dec. 31, 2001). We examined the effect of individual factors, including race, on patient survival using Cox proportional hazards analysis that adjusted only for age as a continuous variable. We then determined whether the effects of race were statistically independent of other factors using Cox proportional hazards analyses that included variables showing an association $(p<0.10)$ with survival in univariate Cox regression analyses. The 
following factors were considered: age, sex, Aboriginal race, initial and subsequent modes of dialysis, socioeconomic status (as IPPE), smoking status, year from initiation of dialysis, community size, dialysis centre (to account for regional differences in clinical practice) and comorbid conditions, including coronary disease (angina, myocardial infarction, previous coronary artery bypass grafting), previous or current hypertension, chronic heart failure, stroke, lung disease, peripheral vascular disease, malignant disease and other serious illness anticipated to significantly reduce life expectancy. Missing comorbidity data (representing about $8 \%$ of all information on comorbidity) was dealt with in 2 ways: by assuming that the characteristic was absent and by using dummy variables to denote missing data. The results did not differ with these 2 methods, so here we have reported results using the latter method. Cross-product interaction terms were used to determine whether the effect of race was modified by age, sex, diabetes or socioeconomic status. In the primary analysis, follow-up was censored at the time of renal transplantation. We also performed a separate Cox regression analysis in which patient follow-up continued after transplantation.

We used additional proportional hazard models to examine the relation between race and likelihood of renal transplantation. We determined that the proportional hazard assumption was satisfied by examining plots of the log-negative-log of the within-group survivorship functions versus log time as well as comparing Kaplan-Meier (observed) with Cox (expected) survival curves. ${ }^{24}$ Statistical significance was defined by $p<0.05$. The study was approved by the institutional review board at the University of Alberta.

\section{Results}

A total of 4840 patients commenced dialysis in Alberta, Saskatchewan and Manitoba during the study period, and for 4333 of these patients, "race" was identified in CORR. Aboriginal patients accounted for 685 (15.8\%) of the study population but $8.7 \%$ of the total population in the catchment area, which suggests that the unadjusted prevalence of ESRD is approximately twice as high among Aboriginal people as among non-Aboriginals. Although the incidence of ESRD increased over the study period for all racial groups (data not shown), the proportion of incident patients who were Aboriginal remained similar over the study period $(p=0.26)$. Of the 4333 incident patients, 510 were of non-Aboriginal, nonwhite race and were excluded from further analysis. The demographic characteristics of the remaining 3823 patients appear in Table 1 . Of these, 16 $(0.4 \%)$ were lost to follow-up, and their data were censored after a median period of 21 days.

Aboriginal patients were younger, less likely to be male and more likely to have diabetes mellitus, chronic heart failure and peripheral vascular disease than white patients (Table 1). Aboriginal patients were less likely to have coronary disease, hypertension or known malignancy at the time of commencing dialysis, less likely to start renal replacement on peritoneal dialysis and more likely to be

Table 2: Factors associated with death and with renal transplantation among patients commencing dialysis treatment between 1990 and 2000*

\begin{tabular}{|c|c|c|c|c|}
\hline \multirow[b]{2}{*}{ Factor } & \multicolumn{2}{|c|}{ Hazard ratio for death (and 95\% Cl) } & \multicolumn{2}{|c|}{ Hazard ratio for transplantation (and 95\% Cl) } \\
\hline & Age-adjusted & Multivariate $†$ & Age-adjusted & Multivariate $\dagger$ \\
\hline Aboriginal race (compared with white race) & $1.15(1.02-1.30)$ & $0.89(0.77-1.02)$ & $0.36 \quad(0.30-0.44)$ & $0.43(0.35-0.53)$ \\
\hline Male sex & $0.99(0.91-1.09)$ & $0.94(0.86-1.03)$ & $1.45 \quad(1.28-1.65)$ & $1.39(1.22-1.58)$ \\
\hline Age (per decade) & $\mathrm{NA}$ & $1.31(1.26-1.36)$ & NA & $0.66(0.64-0.69)$ \\
\hline Lowest quintile of socioeconomic status $\ddagger$ & $0.98(0.88-1.10)$ & $1.00(0.89-1.12)$ & $1.08(0.94-1.24)$ & $1.06(0.92-1.23)$ \\
\hline Diabetes mellitus§ & $1.11(0.94-1.30)$ & $1.21(1.02-1.43)$ & $0.58(0.39-0.88)$ & $0.69(0.45-1.04)$ \\
\hline \multicolumn{5}{|l|}{ Cause of end-stage renal disease } \\
\hline Glomerulonephritis & $1.37(1.00-1.89)$ & $1.38(0.99-1.90)$ & $0.67(0.54-0.84)$ & $0.77(0.61-0.97)$ \\
\hline Diabetic nephropathy & $2.41(1.80-3.23)$ & $2.03(1.51-2.74)$ & $0.35(0.28-0.44)$ & $0.51(0.41-0.65)$ \\
\hline Hypertensive or ischemic renal disease & $2.01(1.48-2.73)$ & $1.72(1.25-2.35)$ & $0.50(0.36-0.68)$ & $0.54(0.40-0.74)$ \\
\hline Other & $1.87(1.39-2.50)$ & $1.66(1.24-2.23)$ & $0.39(0.31-0.48)$ & $0.49(0.39-0.61)$ \\
\hline Coronary disease & $1.45(1.31-1.60)$ & $1.29(1.17-1.44)$ & $0.50(0.41-0.62)$ & $0.62(0.50-0.77)$ \\
\hline Chronic heart failure & $1.66(1.51-1.83)$ & $1.41(1.27-1.56)$ & $0.39(0.32-0.49)$ & $0.52(0.41-0.65)$ \\
\hline Chronic lung disease & $1.32(1.15-1.52)$ & $1.19(1.04-1.38)$ & $0.52(0.37-0.74)$ & $0.60(0.42-0.86)$ \\
\hline Peripheral vascular disease & $1.57(1.41-1.76)$ & $1.42(1.26-1.60)$ & $0.45(0.34-0.61)$ & $0.69(0.51-0.94)$ \\
\hline Malignant disease & $1.26(1.08-1.46)$ & $1.35(1.16-1.58)$ & $0.51(0.33-0.78)$ & $0.49(0.32-0.76)$ \\
\hline Hypertension & $0.87(0.78-0.97)$ & $0.79(0.70-0.88)$ & $1.19(1.03-1.38)$ & $1.21(1.04-1.42)$ \\
\hline Other serious illness & $1.31(1.14-1.50)$ & $1.21(1.04-1.39)$ & $0.45(0.33-0.63)$ & $0.50(0.36-0.69)$ \\
\hline
\end{tabular}

*Follow-up was censored at Dec. 31, 2001, after recovery of renal function, at time of transplantation or on loss to follow-up.

†Adjusted for age, sex, cause of end-stage renal disease, diabetes mellitus, other comorbidity, dialytic modality, dialysis centre, era effect, socioeconomic status and location of residence (urban or rural).

¥Socioeconomic status was defined by neighbourhood income per person equivalent, a measure of household income that is adjusted for household size, on the basis of 1996 Canadian census summary data.

§Patients who had diabetes mellitus but whose end-stage renal disease was not due to diabetic nephropathy.

$\uparrow$ Comparisons with polycystic kidney disease as the cause of end-stage renal disease. 
in the lowest quintile of socioeconomic status.

During the study period, the unadjusted rate of death per 1000 patient-years was $158(95 \%$ confidence interval [CI 144-176) for Aboriginal patients and 146 (95\% CI 139-153) for white patients. The age-adjusted risk of death after initiation of dialysis was significantly higher among Aboriginal than among white patients (hazard ratio [HR] 1.15, 95\% CI 1.02-1.30). The greater age-adjusted risk associated with Aboriginal race was no longer observed after adjustment for the presence or absence of diabetes mellitus (adjusted HR 1.03, 95\% CI 0.91-1.16). Further adjustment for other comorbidity and cause of ESRD further reduced the risk of death associated with Aboriginal race (HR 0.99, 95\% CI 0.87-1.12). In the fully adjusted model, Aboriginal race was associated with an $11 \%$ lower risk of death than white race, although the difference was not statistically significant (HR 0.89, 95\% CI 0.77-1.02) (Table 2, Fig. 1).

The result of a test for interaction was statistically nonsignificant, which suggests that socioeconomic status was associated with mortality to a similar extent among Aboriginals and whites. In addition, there was no evidence that dialysis centre, patient age, patient sex or initial mode of dialysis interacted with the effect of Aboriginal race on mortality, which suggests that the effect of race was homogeneous within the study population.

After initiation of dialysis, Aboriginal patients were significantly less likely to undergo renal transplantation than white patients. During the study period, the crude transplantation rate per 1000 patient-years was $62(95 \%$ CI 52-75) for Aboriginal patients and 133 (95\% CI 125-142) for white patients. Aboriginal patients were significantly less likely than white patients to receive a cadaveric trans-

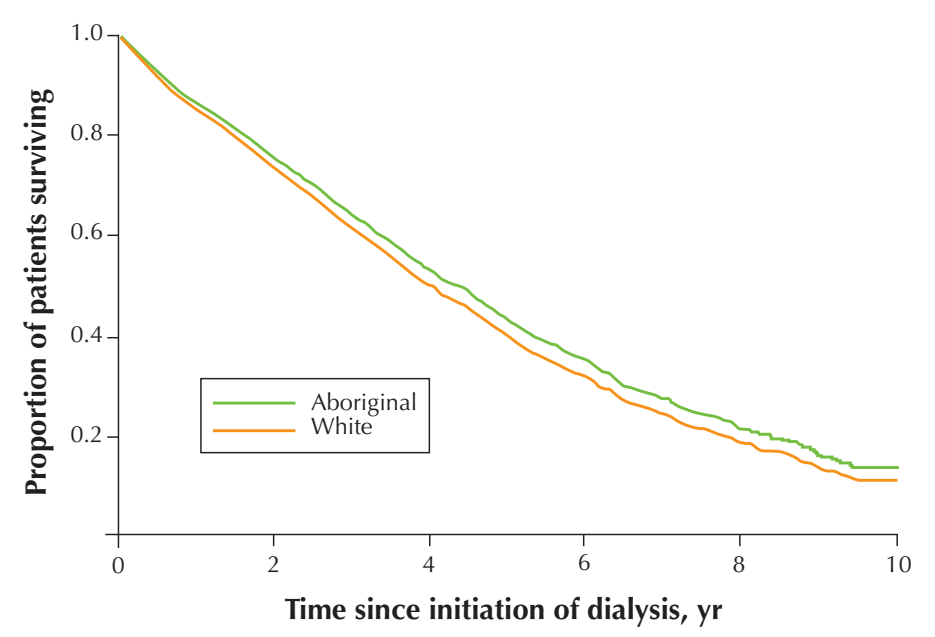

Fig. 1: Adjusted survival of patients, by race. The data have been adjusted for age, sex, cause of end-stage renal disease (ESRD), diabetes mellitus, other comorbidity, mode of dialysis, dialysis centre, era effect, socioeconomic status and location of residence (urban or rural). plant $(12.1 \%$ v. $20.5 \%, p<0.001)$ or a living related transplant $(4.7 \%$ v. $9.2 \%, p<0.001)$.

Using Cox proportional hazard models, we adjusted for other measured variables that might confound the relation between race and transplantation (Table 2). In that analysis, Aboriginal race was independently associated with a reduced likelihood of renal transplantation (HR 0.43, 95\% CI $0.35-0.53)$. The results were similar when patients who received renal transplants from living donors were excluded from the analysis (HR 0.46, 95\% CI $0.36-0.58$ ) or when their data were censored at the time of transplantation (HR 0.48, 95\% CI 0.38-0.61). Adjustment for socioeconomic status did not affect the likelihood of transplantation by race in any of the models (data not shown). In Fig. 2A, the adjusted incidence of transplantation is plotted against time for these 2 groups, stratified by diabetic status.

Because we did not have information on patients' transplant wait-list status, it is possible that comorbidity affected eligibility and thus rates of transplantation. We attempted to address this possibility by performing an additional analysis that considered only younger patients with less comorbidity (age less than 60 years without known coronary disease, chronic heart failure, stroke, chronic lung disease, peripheral vascular disease, known malignant or other serious medical illness that would be expected to reduce life expectancy). In these 1167 patients, and after stratification for diabetic status, Aboriginal race was again independently associated with lower rates of renal transplantation (HR 0.47, 95\% CI 0.37-0.60) (Fig. 2B).

Because renal transplantation is associated with lower rates of death than dialysis, ${ }^{25}$ different rates of transplantation would be expected to influence comparative mortality between racial groups. However, such differences would not be apparent in models that end follow-up at the time of transplantation. To address this issue, we developed additional Cox proportional hazard models for all subjects who commenced renal replacement therapy and included survival after transplantation for those who received renal allografts. In these analyses, the age-adjusted risk of death after initiation of dialysis was higher among Aboriginal patients than white patients (HR 1.36, 95\% CI 1.21-1.52), although the fully adjusted risk of death was similar in the 2 groups (HR 1.02, 95\% CI $0.90-1.16)$. The risk of death associated with Aboriginal race was higher in both analyses that included follow-up after transplantation than in the analyses that ended follow-up at the time of transplantation (HR 1.15 and 0.89 respectively; Table 2). This result suggests that the lower transplantation rate among Aboriginals may adversely affect their survival when receiving renal replacement therapy. 


\section{Interpretation}

Although Aboriginal patients treated with dialysis in the Prairie provinces had higher age-adjusted rates of death than white patients, this difference was no longer observed when the analysis was adjusted for other variables associated with survival, especially diabetes mellitus. Contrary to observations from other populations, ${ }^{12,14}$ the survival of Aboriginals with ESRD appeared to be at least as good as in white patients after adjustment for concomitant illness.

After adjustment for comorbidity, underlying renal disease, location of residence and socioeconomic status, Aboriginal people were less than half as likely to receive renal transplants, which in turn appeared to increase the excess age-adjusted mortality associated with Aboriginal race. This disparity was consistent regardless of donor source (living or cadaveric) and was similar when only young patients without documented comorbidity were considered. Because renal transplantation is associated with lower rates of death, ${ }^{25}$ higher quality of life $\mathrm{e}^{26}$ and lower health care costs than dialysis treatment, the lower transplantation rate among Aboriginal people warrants further investigation.

Our data do not allow us to identify the reasons for the differential access to transplantation by race, but it seems improbable that differences in medical suitability were exclusively responsible. Aboriginal patients might be less likely to be put on waiting lists for transplantation (because of higher rates of comorbidity or perceived poor adherence) or less likely to be matched with a suitable donor (because of lower rates of live kidney donation, less common human leukocyte antigen types or bias in organ allocation). In addition, Aboriginal patients might be less likely to want a renal transplant than white patients, as is the case for other ethnic minorities. ${ }^{27}$ Because of geographic isolation, Aboriginal people may have reduced access to transplantation services or be less likely to attend appointments or procedures aimed at determining suitability for transplantation. However, in the current study, the lower renal transplantation rate among Aboriginal people was independent of residence location, and our study was restricted to regions served by renal transplant programs. Finally, the reduced rates of transplantation among Aboriginals did not vary by dialysis centre. These findings argue against geographic factors as being responsible for differential rates of transplantation by race.

In previous work, referral rates for renal transplant assessment were similar for Native American and white patients residing in Ari- zona and New Mexico, but the delay between starting dialysis and transplantation was significantly longer for the $\mathrm{Na}$ tive Americans. ${ }^{28}$ Similarly, a study conducted in Australia and New Zealand found that indigenous people experienced longer delays than white subjects between starting dialysis and placement on the waiting list for transplantation and between wait-listing and transplantation. ${ }^{5}$ These findings are similar to those observed for black patients treated for ESRD in the United States. ${ }^{29}$

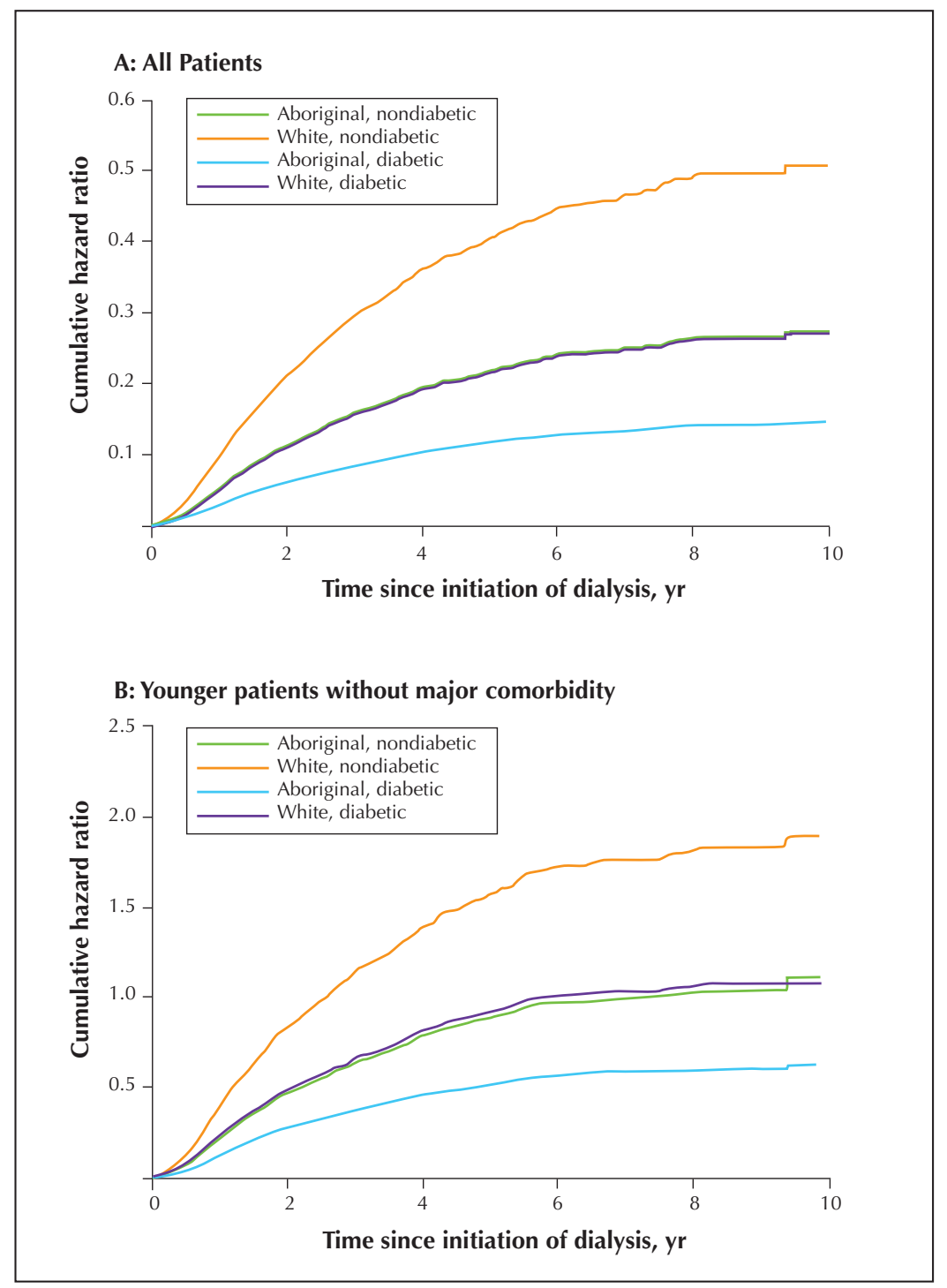

Fig. 2: Likelihood of renal transplantation by race and diabetic status, adjusted for age, sex, cause of ESRD, diabetes mellitus, mode of dialysis, dialysis centre, era effect, socioeconomic status and location of residence (urban or rural), expressed as the cumulative hazard ratio. A: All patients commencing dialysis treatment; these data are also adjusted for comorbidity. B: Younger patients without major comorbidity (age less than 60 years without known coronary disease, chronic heart failure, stroke, chronic lung disease, peripheral vascular disease, malignant or other serious medical illness that would be expected to reduce life expectancy). In both analyses, Aboriginal patients were significantly less likely to receive renal transplants $(p<0.001)$. 
Our study had several limitations. First, referral patterns might have influenced differences in mortality or transplantation rates between the racial groups. We did not have information on the timing of nephrology referral or the level of residual renal function at the time dialysis treatment began. Second, it is possible that bias resulted from the exclusion of patients with unknown racial status. However, it is unlikely that these data would be missing more frequently among Aboriginal patients. Information that was missing or incorrect at random would bias our results toward the null, and repeating our analyses classifying all people of unknown race as white did not change any of the findings (data not shown). Similarly, racial status was provided to CORR by dialysis centre personnel, rather than the subjects themselves, and race might therefore have been incorrectly classified in some cases. Although we cannot exclude this possibility, it is unlikely that differential misclassification occurred by race. Again, this suggests that any resulting bias would reduce the statistical power rather than result in spurious findings. Finally, our analyses are limited by the lack of data on transplant wait-list status, which might help to explain how the markedly lower renal transplantation rates among Aboriginal patients could be increased.

In conclusion, we found that Aboriginal patients treated for ESRD in the Prairie provinces had more comorbid illness at initiation of dialysis than white patients. Once established on renal replacement, such patients had higher age-adjusted rates of death, but after further adjustment for diabetes mellitus and other comorbid illness, rates of death were similar for Aboriginal and white patients. However, Aboriginal patients had lower rates of renal transplantation than non-Aboriginals, independent of measured comorbidity and socioeconomic and geographic factors. These findings require further investigation.

\section{This article has been peer reviewed.}

From the Department of Medicine, Division of Nephrology (Tonelli, Gourishankar), the Division of Critical Care Medicine (Tonelli) University of Alberta, Edmonton, Alta.; the Institute of Health Economics, Edmonton, Alta. (Tonelli, Manns); the Department of Community Health Sciences (Hemmelgarn, Manns) and the Department of Medicine, Division of Nephrology (Manns), University of Calgary, Calgary, Alta., the Department of Medicine, Division of Nephrology, University of Saskatchewan, Saskatoon, Sask. (Pylypchuk); the Department of Medicine, Section of Nephrology, Winnipeg Health Sciences Centre, Winnipeg, Man. (Bohm); the Department of Medicine, Division of Nephrology, Queen's University, Kingston, Ont. (Yeates); the Division of Nephrology, St. Paul's Hospital, Vancouver, BC (Gill); and the Division of Nephrology, Tufts-New England Medical Center, Boston, Mass. (Gill)

\section{Competing interests: None declared.}

Contributors: Drs. Tonelli, Gill and Hemmelgarn were responsible for study conception and design. All authors participated in the analysis and interpretation of the data. Dr. Tonelli drafted the article, and all authors helped to revise the article for important intellectual content and approved the final version for publication.

Acknowledgements: We thank Frank Ivis and Kim Badovinac of the Canadian Organ Replacement Register for their assistance in facilitating our data request. Grant support for this work was received from the Alberta Heritage Foundation for Medical Research (through Dr. Tonelli), the Canadian Institutes of Health Research (through Dr. Manns) and the Kidney Foundation of Canada (through Dr. Gill). CORR is funded by Health Canada (15\%) and the provincial and territorial departments of health (80\%) on the basis of population. The Kidney Foundation of Canada and the health care industry provide the remaining funding (approximately $5 \%$ ).

\section{References}

1. Burrows NR, Geiss LS, Engelgau MM, Acton KJ. Prevalence of diabetes among Native Americans and Alaska Natives, 1990-1997: an increasing burden. Diabetes Care 2000;23(12):1786-90.

2. Fagot-Campagna A, Pettitt DJ, Engelgau MM, Burrows NR, Geiss LS, Valdez R, et al. Type 2 diabetes among North American children and adolescents: an epidemiologic review and a public health perspective. 7 Pediatr 2000;136(5):664-72.

3. Dean HJ, Mundy RL, Moffatt M. Non-insulin-dependent diabetes mellitus in Indian children in Manitoba. CMA7 1992;147(1):52-7.

4. Daniel M, Rowley KG, McDermott R, Mylvaganam A, O'Dea K. Diabetes incidence in an Australian aboriginal population. An 8-year follow-up study. Diabetes Care 1999;22(12):1993-8

5. McDonald SP, Russ GR. Burden of end-stage renal disease among indigenous peoples in Australia and New Zealand. Kidney Int Suppl 2003;(83):S123-7.

6. Narva AS. The spectrum of kidney disease in American Indians. Kidney Int Suppl 2003;(83):S3-7.

7. Dyck RF, Tan L. Rates and outcomes of diabetic end-stage renal disease among registered native people in Saskatchewan. CMA7 1994;150(2):203-8.

8. Dyck RF. Mechanisms of renal disease in indigenous populations: influences at work in Canadian indigenous peoples. Nephrology 2001;6:3-7.

9. Dean HJ, Young TK, Flett B, Wood-Steiman P. Screening for type-2 diabetes in aboriginal children in northern Canada. Lancet 1998;352(9139):1523-4.

10. Green C, Blanchard JF, Young TK, Griffith J. The epidemiology of diabetes in the Manitoba-registered First Nation population: current patterns and comparative trends. Diabetes Care 2003;26(7):1993-8.

11. National Steering Committee of the First Nations and Inuit Regional Health Survey. Final report. Ottawa: The Committee; 1999.

12. Statistics Canada. 1996 census handbook. Ottawa: Industry Canada; 1997. Cat no 92-352-XPE.

13. Callaghan RC. Risk factors associated with dropout and readmission among First Nations individuals admitted to an inpatient alcohol and drug detoxification program. CMA7 2003;169(1):23-7.

14. Injury mortality among American Indian and Alaska Native children and youth United States, 1989-1998. MMWR Morb Mortal Wkly Rep 2003;52(30):697-701.

15. Stephens D. Critical illness and its impact on the Aboriginal people of the top end of the Northern Territory, Australia. Anaesth Intensive Care 2003;31(3):294-9.

16. Anand SS, Yusuf S, Jacobs R, Davis AD, Yi Q, Gerstein H, et al. Risk factors, atherosclerosis, and cardiovascular disease among Aboriginal people in $\mathrm{Ca}$ nada: the Study of Health Assessment and Risk Evaluation in Aboriginal Peoples (SHARE-AP). Lancet 2001;358(9288):1147-53.

17. Shah BR, Gunraj N, Hux JE. Markers of access to and quality of primary care for aboriginal people in Ontario, Canada. Am 7 Public Health 2003;93(5):798-802

18. Ellerby JH, McKenzie J, McKay S, Gariepy GJ, Kaufert JM. Bioethics for clinicians: 18. Aboriginal cultures. CMA7 2000;163(7):845-50.

19. Powe NR. To have and have not: health and health care disparities in chronic kidney disease. Kidney Int 2003;64(2):763-72.

20. Canadian Organ Replacement Registry report: dialysis and renal transplantation. Vol 1. Ottawa: The Registry; 1998.

21. Schaubel DE, Stewart DE, Morrison HI, Zimmerman DL, Cameron JI, Jeffery JJ, et al. Sex inequality in kidney transplantation rates. Arch Intern Med 2000;160(15):2349-54

22. Pei YP, Greenwood CM, Chery AL, Wu GG. Racial differences in survival of patients on dialysis. Kidney Int 2000;58(3):1293-9.

23. $\mathrm{Ng}$ E, Wilkins R, Perras A. How far is it to the nearest hospital? Calculating distances using the Statistics Canada postal code conversion file. Health Rep 1993;5(2):179-88.

24. Hosmer DW, Lemeshow S. Applied survival analysis. Regression modeling of time to event data. New York: John Wiley and Sons; 1999.

25. Wolfe RA, Ashby VB, Milford EL, Ojo AO, Ettenger RE, Agodoa LY, et al. Comparison of mortality in all patients on dialysis, patients on dialysis awaiting transplantation, and recipients of a first cadaveric transplant. $N$ Engl 7 Med 1999;341(23):1725-30.

26. Laupacis A, Keown P, Pus N, Krueger H, Ferguson B, Wong C, et al. A study of the quality of life and cost-utility of renal transplantation. Kidney Int 1996;50(1):235-42.

27. Ettinger B. Recurrent nephrolithiasis: natural history and effect of phosphate therapy. A double-blind controlled study. Am f Med 1976;61(2):200-6.

28. Narva A, Stiles S, Karp S, Turak A. Access of Native Americans to renal transplantation in Arizona and New Mexico. Blood Purif 1996;14(4):293-304.

29. Epstein AM, Ayanian JZ, Keogh JH, Noonan SJ, Armistead N, Cleary PD, et al. Racial disparities in access to renal transplantation - Clinically appropriate or due to underuse or overuse? NEngl 7 Med 2000;343(21):1537-44, 2 p preceding 1537.

Correspondence to: Dr. Marcello Tonelli, Division of Nephrology, University of Alberta, 7-129 Clinical Science Bldg. 8440112 St., Edmonton AB T6G 2G3; fax 780 407-7878; mtonelli@ualberta.ca 